\title{
Magnetic Resonance Force Microscopy With a Ferromagnetic Tip Mounted on the Force Detector
}

\author{
Z. Zhang \\ Condensed Matter and Thermal Physics Group and Center for Nonlinear Studies \\ Los Alamos National Laboratory, Los Alamos, NM 87545 \\ P. C. Hammel \\ Condensed Matter and Thermal Physics Group \\ Los Alamos National Laboratory, Los Alamos, NM 87545 \\ (Version date: October 8, 1997)
}

\begin{abstract}
The Magnetic Resonance Force Microscope (MRFM) presents the opportunity for a magnetic resonance imaging probe with ultra-high, potentially atomic-scale, resolution. The successful application of this technique in detection of nuclear magnetic, electron-spin and ferromagnetic resonance (FMR) highlights its significant potential. We discuss the capabilities of the MRFM with particular emphasis on the detection of FMR using MRFM techniques. A crucial remaining challenge in the development of the magnetic resonance force microscope (MRFM) is to place the magnetic probe on the mechanical resonator. We address the problem of spurious detector response arising from interactions between the magnetic tip and various external applied fields. We show that miniature, magnetically-polarized $\mathrm{Nd}_{2} \mathrm{Fe}_{14} \mathrm{~B}$ particles show promise as magnetic probe tips. Our experience indicates it will be important to minimize the total polarized moment of the magnetic tip and to ensure that the applied fields are as uniform as possible.
\end{abstract}

Key Words: magnetic resonance force microscopy, mechanical detection, cantilever, force microscopy, electron spin resonance, nuclear magnetic resonance, ferromagnetic resonance, magnetic resonance imaging. 


\section{INTRODUCTION}

The magnetic resonance force microscope (MRFM) is a microscopic, three-dimensional imaging technique with the potential for achieving atomic scale resolution $[1,2]$. MRFM employs mechanical detection of the magnetic resonance signal in place of inductive detection used in conventional magnetic resonance imaging (MRI). The ultra-high force sensitivity of the resonator (demonstrated in the atomic force microscope) enables much higher sensitivity to spin magnetization in MRFM and thus much higher spatial resolution than is achievable in conventional MRI. MRFM has been successfully demonstrated in electron spin resonance (ESR) [3-7], ferromagnetic resonance (FMR) [8], and nuclear magnetic resonance (NMR) $[9,10]$. For instance, spatial resolution of $\sim 1 \mu \mathrm{m}$ has been achieved in NMR $[9,10]$, already an order of magnitude better than conventional MRI. Further increase of the sensitivity and resolution can be achieved by performing MRFM at cryogenic temperatures, by increasing the field gradient and by improving the performance of the micro-mechanical resonators [11].

In order to apply MRFM to samples of arbitrary size and shape, the magnetic tip, which generates the necessary field gradient and the force between the resonator and the sample,

must be placed on the resonator (i.e., in a "scanning probe geometry"). One of the primary impediments to implementing the scanning probe geometry is the large, spurious forces on the sensitive force detector which will arise from interactions between the magnetic tip and various time-varying applied magnetic fields which can mask the desired resonance signal. Here we discuss some of the problems we have encountered in our exploration of this goal and some solutions to these problems.

\section{THE MAGNETIC RESONANCE FORCE MICROSCOPE}

A schematic illustration of a MRFM setup is shown in Fig. 1. It includes a $\mathrm{Nd}_{2} \mathrm{Fe}_{14} \mathrm{~B}$ bar magnet $0.56 \mathrm{~mm}$ diameter by $6.35 \mathrm{~mm}$ long mounted on a translatable stage which provides both the necessary field gradient $\nabla \mathbf{B}$ and an average magnetic field $B_{\mathrm{bar}} \hat{\mathbf{z}}$ (see Ref. 
[6] for more detail concerning the bar magnet); an electromagnet which provides a ramped bias field $B_{0} \hat{\mathbf{z}}$; and a modulation coil [12] which produces a field $\mathbf{B}_{m}(t)=B_{m} \sin \left(2 \pi f_{m} t\right) \hat{\mathbf{z}}$. The total magnetic field at the sample is $\mathbf{B}=\left[B_{\mathrm{bar}}+B_{0}+B_{m}\right] \hat{\mathbf{z}}$. Here, we neglect the small field gradients perpendicular to $\hat{\mathbf{z}}$, a good approximation given the small size (of order $10 \mu \mathrm{m})$ of the sample relative to the bar magnet $(\sim 0.5 \mathrm{~mm})$. An rf field $B_{\mathrm{rf}}(t)=B_{1} e^{i 2 \pi f_{\mathrm{rf}} t}$ oriented perpendicular to $\hat{\mathbf{z}}\left(f_{\mathrm{rf}}=500-1000 \mathrm{MHz}\right)$ is produced by a coil placed near the sample mounted on an AFM cantilever. The spins in the sample produce a moment $\mathbf{M}$ which interacts with the field gradient producing a force on the cantilever

$$
\mathbf{F}=\mathbf{M} \cdot \nabla \mathbf{B}=M_{z} \frac{\partial B}{\partial z} \hat{\mathbf{z}}
$$

where $\hat{\mathbf{z}}$ is the unit vector parallel to the bias field and/or the relevant cantilever vibration direction. Because of the field gradient $\partial B / \partial z$, a shell ("sensitive slice") of constant field exists within which the magnetic resonance condition, $f_{\mathrm{rf}}=f_{\mathrm{res}}(B)$ is met. Here, $f_{\mathrm{res}}(B)$ is determined by the interaction of the moments with the applied field and with the other moments; for non-interacting moments $2 \pi f_{\text {res }}(B)=\gamma B$, where $\gamma$ is the gyromagnetic ratio. In the case of FMR where the interactions amongst moments are strong, the expression is less simple [13]. The position of this sensitive slice moves as the bias field is ramped. If the slice intersects the sample, saturation [14] of the magnetization in this slice by the applied rf field suppresses the net magnetic moment along the $z$-direction thus changing the force on the cantilever.

The technique of mechanical detection of magnetic resonance relies on manipulating the spin magnetization $M_{z}$ in the sensitive slice such that the force $F(t)=M_{z}(t) \partial B / \partial z$ varies at $f_{c}$, thus driving the mechanical resonator at its resonance frequency $f_{c}$. Modulation of either the bias field $B_{0}$, the rf field amplitude $B_{1}$ or the rf frequency $f_{\mathrm{rf}}$ will cause periodic saturation and thus a modulation of $M_{z}$. In order to reduce the spurious response that can arise with these modulations, a more complex anharmonic modulation technique [5] was developed in which $B_{0}$ is modulated at $f_{m}$ and $\left|B_{1}\right|^{2}$ at $f_{1}$ such that $\left|f_{1} \pm f_{m}\right|=f_{c}$. Due to the nonlinear response of $M_{z}$ at resonance, the frequency spectrum of $M_{z}(t)$ will have a 
component at $f_{c}$; this produces the desired driving force.

The force is detected by measuring the magnitude and phase of the cantilever oscillation $f_{c}$ using an optical fiber interferometer and a lock-in amplifier. We presently use commercially available atomic force microscope cantilevers [15] (i.e., clamped on one end only) with force constants $k \sim 0.08 \mathrm{~N} / \mathrm{m}$ and (unloaded) resonant frequencies $f_{c} \sim 6-20 \mathrm{kHz}$. A spectrum is obtained by plotting the magnitude of the oscillation in-phase with a reference signal as a function of applied bias field $B$; a negative magnitude indicates a phase shift of $\pi$ between the resonator and the reference. All experiments to date have been performed with small $(\sim 2-20$ nanogram $)$ samples mounted on the cantilever tip. This mass reduces the resonant frequency and $Q$ of the resonator. The $Q$ of loaded cantilevers can be as high as 30,000 in vacuum at room temperature. The oscillation of the cantilever is detected by means of a fiber-optic, diode laser interferometer.

A typical electron spin resonance MRFM spectrum from a sample of DPPH $(2,2-$ diphenyl-1-picrylhydrazyl) is shown in Fig. 2. In this particular experiment, two DPPH particles are mounted on the cantilever as shown in the inset of Fig. 2. The thermal displacement noise of the cantilever is visible $(\sim 1 \AA)$; the sensitivity of this measurement is discussed in detail in Ref. [7]. The uncertainty in determining the distance between the centers of the two particles, a measure of the spatial resolution in this direction, is about $1 \mu \mathrm{m}$ in this case. This figure illustrates the capability of sub-surface imaging of MRFM.

\section{MICROSCOPIC FERROMAGNETIC RESONANCE}

There are two motivations for performing FMR experiments using the MRFM. First

is the tremendous promise of microscopic FMR experiments for elucidating the nature of scientifically and technologically important magnetic materials such as magnetic multilayer systems displaying giant magnetoresistance (GMR). It is important to understand how the performance of such devices is influenced by microscopic spatial variations in properties like interface quality, exchange coupling and magnetic anisotropy. Conventional FMR has proved 
to be a powerful technique in evaluating the average values of these properties within the sample. With the addition of the microscopic imaging capability of MRFM, FMR/MRFM could become one of the primary characterization techniques for such devices. Second, these experiment provides us an opportunity to study the spurious couplings which arise in a scanning geometry due to the strong interactions between applied time-varying fields and a ferromagnetic tip mounted on the cantilever.

Fig. 3 shows a typical MRFM spectrum of a microscopic single crystal yttrium iron garnet (YIG) thin film (about $20 \times 40 \times 3 \mu \mathrm{m}^{3}$ but having an irregular shape). YIG is a ferrimagnetic material having a net magnetization of $140 \mathrm{emu} / \mathrm{cm}^{3}$ at room temperature. We were required to damp the cantilever oscillation by performing our MRFM experiment at ambient pressure so as to avoid excessively large oscillation amplitudes. Dramatic improvements in signal to noise ratio $(S / N \propto \sqrt{Q}$, where $Q$ is the resonator quality factor [16]) will be obtained by reducing pressure (which increases the $Q$ of the cantilever by three orders of magnitude) will enable the detection of signals from much smaller sample volumes.

The strongest signal, observed at a field of $\sim 100 \mathrm{G}$, displayed an extremely asymmetric derivative lineshape and the resonance field was independent of the applied rf frequency, indicating that this mode is not related to the resonance behavior of the spins in the YIG sample. In fact, this signal can be observed even in the absence of any rf field when the bias field is modulated at $f_{c} / 2$ (to reduce direct coupling between the cantilever and the modulation field). From Eq. 1 it can be seen that the MRFM experiment is sensitive to any variation in the component of the magnetic moment parallel to the field gradient. In particular it will be sensitive to "resonance modes" which arise as a consequence of resonant coupling of the rf excitation to the spins in the $\hat{\mathbf{x}}-\hat{\mathbf{y}}$ plane, or "non-resonance" (NR) modes which, in this case, are due to the change of the orientation of the sample magnetization in response to variations of the bias field. Given the dimensions of the YIG sample, we calculated the internal demagnetization field within the film. Based on this we found [8] that the NR mode is strongest when the magnitude of the applied field is close to but less than the value necessary to saturate the magnetization in the center of the film. In this 
field range, a small field modulation will cause a substantial change in the saturated volume of the sample; this leads to a large variation in the magnetized moment along the applied field orientation. This result indicates that in order to reduce the spurious coupling it is important that the magnetized moment of the tip used in the scanning probe geometry be independent of the applied bias field, at least in the field range where the resonance slice intersects the sample.

We turn now to the resonance mode we observe. On the high field side of the NR mode, we observe a family of genuine resonance modes whose elements we label $m_{1}, m_{2}, \ldots$ (Fig. 3). With increasing rf frequency, the entire family of modes moves to higher field, away from the nonresonance (NR) mode. These are magneto-static modes first calculated by Damon and Eshbach (DE) [17]. The field position of the first resonance mode $m_{1}$ reflects the magnetic anisotropy energy of the sample while the separation between any two modes depends on the magnetic exchange coupling as well as the physical shape of the YIG film. The application of DE theory in our case is complicated by the small size and irregular shape of our sample and by the fact that the sample is not fully saturated at resonance. Although quantitative evaluation is difficult, our results agree qualitatively with the estimates based on DE theory in a rectangularly-shaped, saturated YIG medium.

The strength of the FMR signals we obtain from the microscopic sample of single crystal YIG film indicate that MRFM has the potential to study a large variety of magnetic materials with very high sensitivity. The $S / N$ of the spectrum in Fig. 3 is sufficiently high that thermal noise of the cantilever is not visible; all of the features of the signal are reproduced upon repeating the measurement [8]. By increasing the field gradient associated with the probe magnet, we expect it will be possible to conduct microscopic FMR experiments with micron or sub-micron resolution. However, the existence of the NR mode means that the experimental arrangement must be carefully selected so that the resonance modes and the NR mode occur at well separated fields. Two important issues in this regard are the orientation of the sample with respect to the bias field and the frequency of the rf field. The results shown in Fig. 3 were obtained with the bias field applied in the plane of the YIG 
film.

\section{MRFM WITH A PERMANENT MAGNET ON THE CANTILEVER}

Soft magnetic thin films are commonly used as magnetic recording head in the magnetic information storage industry. It is natural to consider them as candidates for the magnetic tip in the MRFM setup. However, for a typical rf resonance frequency $f_{\mathrm{rf}}$ of $\leq 1 \mathrm{GHz}$, the resonance field $2 \pi f_{\mathrm{rf}} / \gamma\left(\gamma=1.76 \times 10^{7}(\mathrm{Gs})^{-1}\right)$ for free electrons is only a few hundred Gauss. Within this field range, the static magnetic polarization of the magnetic films could change dramatically in response to changing bias field, thus creating a large spurious coupling of the various applied fields to the resonator. We have chosen, then, to explore the possibility of using a magnetic material with a large magnetic anisotropy in the hope this would prevent its magnetization from changing in response to an external field varying over a range of several hundred gauss. To this end, we have explored the characteristics of an MRFM employing a cantilever with a fine particle of $\mathrm{Nd}_{2} \mathrm{Fe}_{14} \mathrm{~B}$ attached to serve as a magnetic tip. The particle [18] was placed on a cantilever in an epoxy which sets in several hours. The cantilever was then placed in a $4.2 \mathrm{~T}$ magnetic field oriented parallel to the long axis of the cantilever while the epoxy was setting. The shape of the glued sample is irregular, but close to elliptical with approximate dimensions $40 \times 40 \times 70 \mu \mathrm{m}^{3}$.

As there is no data for the magnitude of the magnetic field of $\mathrm{Nd}_{2} \mathrm{Fe}_{14} \mathrm{~B}$ micro-particles, we measured the magnetic field $B_{M} \hat{\mathbf{z}}$ generated by this $\mathrm{Nd}_{2} \mathrm{Fe}_{14} \mathrm{~B}$ particle by performing an ESR/MRFM experiment to measure the field generated by the particle at the site of a DPPH particle. The setup is similar to that shown in Fig. 1 except that the bar magnet is now replaced by the magnetized $\mathrm{Nd}_{2} \mathrm{Fe}_{14} \mathrm{~B}$ particle with its polarization axis parallel to the $z$-axis. At a given $\mathrm{rf}$ frequency $f_{\mathrm{rf}}$, the magnetic field $B_{0}+B_{M}$ at the site of the DPPH particle is constant at $2 \pi f_{\mathrm{rf}} / \gamma$. The variation of $B_{M}$ with the distance $z$ between centers of the DPPH particle and the $\mathrm{Nd}_{2} \mathrm{Fe}_{14} \mathrm{~B}$ particle can be obtained by moving the $\mathrm{Nd}_{2} \mathrm{Fe}_{14} \mathrm{~B}$ particle along the $z$-axis. The results of such a measurement are shown in Fig. 4 for $\mathbf{B}_{0}$ 
both parallel and antiparallel to the initial polarization direction of the $\mathrm{Nd}_{2} \mathrm{Fe}_{14} \mathrm{~B}$ particle. From this measurement we see that the polarized magnetization of the $\mathrm{Nd}_{2} \mathrm{Fe}_{14} \mathrm{~B}$ particle does not reverse its orientation as the applied field is reversed. Because the variation of $B_{M}$ with $z$ depends not only on the polarized magnetic moment $M_{H}$ of the $\mathrm{Nd}_{2} \mathrm{Fe}_{14} \mathrm{~B}$ particle but also on the physical shape of the particle, an accurate evaluation of $M_{H}$ is impossible without the knowledge of the actual particle shape. However, regardless of shape assumed, we find that $M_{H}$ is about $25 \%$ smaller in magnitude when $\mathbf{B}_{0} \|-\mathbf{M}$ (where $B_{0} \sim 300 \mathrm{G}$ ) than that when $\mathbf{B}_{0} \| \mathbf{M}$. This indicates that $M_{H}$ still varies with applied field, but that the variation is much smaller than that in the YIG thin film. We can expect, then, that this magnetic tip will have a smaller spurious coupling than other soft, thin-film magnets in a scanning geometry. On the other hand, use of $\mathrm{Nd}_{2} \mathrm{Fe}_{14} \mathrm{~B}$ particles fabricated so as to have larger coercivities would be desirable in this regard. If we assume a cylindrical shape with a radius of $20 \mu \mathrm{m}$ and a length of $70 \mu \mathrm{m}$ for the particle, we can calculate the dependence of $B_{M}$ on $z$ using the polarized magnetization $\mathcal{M}_{H}$ of the $\mathrm{Nd}_{2} \mathrm{Fe}_{14} \mathrm{~B}$ particle as the only adjustable parameter. The results of the fit are shown by the solid lines in Fig. 4. We find the remanent magnetization $\mathcal{M}_{r} \sim 100 \mathrm{emu} / \mathrm{cm}^{3}$, an order of magnitude smaller than the bulk value.

In principle, by monitoring the motion of this cantilever, we are now in a position to perform MRFM in the scanning probe geometry. No strong NR mode exists in the MRFM spectrum as expected. However, we find that the resonance frequency $f_{c}$ of the cantilever varies as the bias field $B_{0}$ is ramped (shown in Fig. 5). This makes it difficult to modulate the spin magnetization of the sample at $f_{c}$ in the MRFM experiment. The origin of this effect is in the interaction between the applied field and the spin magnetization of the $\mathrm{Nd}_{2} \mathrm{Fe}_{14} \mathrm{~B}$ particles mounted on the cantilever. Two different interaction mechanisms exist in this system. The dominant factor is the torque $\mathbf{M}_{H} \times \mathbf{B}_{0}=M_{H} B_{0} \sin \theta$ on the cantilever when the cantilever is at an angle $\theta$ with respect to the bias field $B_{0}$. Here we assume that the net magnetic moment is always parallel to the long dimension of the cantilever. This leads to a restoring force $F_{\tau}$ at the end of the cantilever in addition to the flexural response of the 
cantilever as shown in Fig. 6a:

$$
F_{\tau}=\frac{M_{H} B_{0}}{L} \sin \theta
$$

where $L$ is the effective length of the cantilever. In our arrangement (shown in Fig. 1) the electromagnet which produces $B_{0}$, also generates a field gradient $\partial B_{0} / \partial z=-\alpha B_{0}$ along the polarization axis of the $\mathrm{Nd}_{2} \mathrm{Fe}_{14} \mathrm{~B}$ particle, where $\alpha$ is a constant that depends on the position of the $\mathrm{Nd}_{2} \mathrm{Fe}_{14} \mathrm{~B}$ magnet in the field of the electromagnet. The interaction between this gradient and the (very large) magnetic moment $M_{H}$ of the $\mathrm{Nd}_{2} \mathrm{Fe}_{14} \mathrm{~B}$ particle also adds to the restoring force:

$$
\mathbf{F}_{g}=M_{H} \frac{\partial B_{0}}{\partial z} \hat{\mathbf{z}}=-\alpha M_{H} B_{0} \hat{\mathbf{z}}
$$

when the cantilever deviates from its equilibrium position (also shown in Fig. 6a).

The simplified model shown in Fig. 6b, predicts the following relation between $f_{c}$ and $B_{0}:$

$$
\begin{aligned}
f_{c} & =f_{c}^{0} \sqrt{1+(1-\alpha L) \frac{M_{H} B_{0}}{k L^{2}}} \\
& \approx f_{c}^{0}\left[1+(1-\alpha L) \frac{M_{H} B_{0}}{2 k L^{2}}\right]
\end{aligned}
$$

where $f_{c}^{0}$ is the cantilever resonance frequency in zero field, $k$ is the spring constant. We assume here that $M_{H} B_{0} / 2 k L^{2} \ll 1$ and $\alpha L<1$. For the measurement shown in Fig. $5, \alpha$ is about $7.5 \times 10^{-5}(\mu \mathrm{m})^{-1}$ measured from a separate experiment; setting $L$ equal to the total length of the cantilever $(440 \mu \mathrm{m})$, we have $\alpha L \ll 1$. In agreement with the data, Eq. 4 predicts that $f_{c}$ will increase with increasing bias field parallel to the polarization direction of the $\mathrm{Nd}_{2} \mathrm{Fe}_{14} \mathrm{~B}$ particle. The slight upward curvature of the $f_{c}$ dependence on $B_{0}$ also supports the earlier conclusion that the magnetized moment of the $\mathrm{Nd}_{2} \mathrm{Fe}_{14} \mathrm{~B}$ particle increases slightly with $B_{0}$ over this field range. Using $k=0.08 \mathrm{~N} / \mathrm{m}$ (given by the manufacturer) and the slope obtained from Fig. 5, the estimated polarized moment $M_{H}$ is $\sim 0.5 \times 10^{-5}$ emu which is equivalent to a remanent magnetization $\mathcal{M}_{r}$ of $55 \mathrm{emu} / \mathrm{cm}^{3}$ for the cylindrical particle we assumed in the previous field measurement. 
The discrepancy between this number and the evaluation of $\mathcal{M}_{r} \sim 100 \mathrm{emu} / \mathrm{cm}^{3}$ from the field measurement is reasonable given the rough approximations in our models and the uncertainties in properties like $k, L$, the volume and shape of the $\mathrm{Nd}_{2} \mathrm{Fe}_{14} \mathrm{~B}$ particle. For instance, the field measurement is sensitive to the physical shape of the $\mathrm{Nd}_{2} \mathrm{Fe}_{14} \mathrm{~B}$ particle and the position of the $\mathrm{DPPH}$ relative to the $\mathrm{Nd}_{2} \mathrm{Fe}_{14} \mathrm{~B}$ particle while the frequency drift we measure depends only on the total magnetic moment of the particle. In addition, $k$ is uncertain by as much as a factor of 3 based on the manufacturer's data, and the effective length $L$ will in fact be $\sim 75 \%$ of the actual cantilever length since the cantilever is not able to rotate freely about its base. Finally, when the cantilever deviates from its equilibrium position the net magnetic moment of the $\mathrm{Nd}_{2} \mathrm{Fe}_{14} \mathrm{~B}$ particle will not remain exactly parallel to the cantilever. Instead, it will rotate away from the cantilever orientation towards the bias field direction. As a result, our estimate of $M_{H}$ from Eq. 4 will be smaller than its actual value.

Our results clearly indicate that the frequency drift of the cantilever is directly related to the interaction between the magnetic tip and the bias field in the scanning geometry. If we require that the frequency drift $\Delta f_{c}$ during a field scan of width $\Delta B_{0}$ be less than the frequency width of the cantilever's mechanical resonance: $\Delta f_{c}<f_{c} / Q$, we can estimate the maximum allowable magnetic moment $M_{H}$ for a given MRFM experiment:

$$
M_{H}<\frac{2 k L^{2}}{Q \Delta B_{0}}
$$

Given $k=0.08 \mathrm{~N} / \mathrm{m}, L=440 \mu \mathrm{m}, \Delta B_{0}=1 \mathrm{kG}$ and $Q=15,000, M_{H}$ should be smaller than $2 \times 10^{-8}$ emu for a $\mathrm{Nd}_{2} \mathrm{Fe}_{14} \mathrm{~B}$ magnet. Note that for magnetic tips with small shape and crystal anisotropies, $M_{H}$ could be significantly larger than this. In the case where the condition in Eq. 5 is met, the field gradient of the bias field could become dominant and the uniformity of the applied field will be an important consideration in designing a MRFM apparatus in the scanning geometry. 


\section{CONCLUSIONS}

The broad applicability of the MRFM has been demonstrated in several experiments. Successful application to FMR measurements of microscopic ferromagnets indicates its significant potential in microscopic studies of multilayer magnetic materials. Three requirements are essential to perform MRFM with the magnetic tip mounted on the mechanical resonator. First, the polarized magnetic moment of the tip must be independent of the ap-

plied bias field in the field range where magnetic resonance occurs. Secondly, the magnetic tip must have small shape and crystal anisotropies to reduce the torque on the cantilever. Thirdly, the bias field itself must be as uniform as possible to minimize the force between the magnetic tip and gradients in the bias field.

We find, as expected, that the coercive field and remanent magnetization of $\mathrm{Nd}_{2} \mathrm{Fe}_{14} \mathrm{~B}$ micro-particles are significantly reduced from their bulk values. They have much smaller non-resonance signals than most soft magnetic thin films in the field range of a few hundred gauss. However due to its large anisotropy energy, the orientation of the $\mathrm{Nd}_{2} \mathrm{Fe}_{14} \mathrm{~B}$ magnetic moment with respect to the applied field will change as the cantilever flexes. The interaction of this moment with the field causes the restoring force and thus the cantilever resonant frequency to be field dependent. This undesirable effect will limit the amount of $\mathrm{Nd}_{2} \mathrm{Fe}_{14} \mathrm{~B}$ that can be placed on the cantilever.

\section{ACKNOWLEDGMENTS}

We gratefully acknowledge fruitful discussions with R. Schwarz in the course of performing this work. We thank R. W. McCallum for the $\mathrm{Nd}_{2} \mathrm{Fe}_{14} \mathrm{~B}$ ingot and R. Schwarz for preparing the powder from the ingot. Work at Los Alamos National Laboratory is performed under the auspices of the United States Department of Energy. 


\section{REFERENCES}

[1] J. A. Sidles, Appl. Phys. Lett. 58, 2854 (1991).

[2] J. A. Sidles, Phys. Rev. Lett. 68, 1124 (1992).

[3] D. Rugar, C. S. Yannoni, and J. A. Sidles, Nature 360, 563 (1992).

[4] O. Züger and D. Rugar, Appl. Phys. Lett. 63, 2496 (1993).

[5] K. J. Bruland, J. Krzystek, J. L. Garbini, and J. A. Sidles, Rev. Sci. Instr. 66, 2853 (1995).

[6] P. C. Hammel, Z. Zhang, G. J. Moore, and M. L. Roukes, J. Low Temp. Phys. 101, 59 (1995).

[7] Z. Zhang, M. L. Roukes, and P. C. Hammel, J. Appl. Phys. 80, 6931 (1996).

[8] Z. Zhang, P. C. Hammel, and P. E. Wigen, Appl. Phys. Lett. 68, 2005 (1996).

[9] D. Rugar et al., Science 264, 1560 (1994).

[10] O. Züger, S. T. Hoen, C. S. Yannoni, and D. Rugar, J. Appl. Phys. 79, 1881 (1996).

[11] J. A. Sidles et al., Rev. Mod. Phys. 67, 249 (1995).

[12] The modulation coil is approximately $1.2 \mathrm{~cm} \mathrm{ID,} 2.5 \mathrm{~cm}$ OD and $0.6 \mathrm{~cm}$ long and located approximately $1 \mathrm{~cm}$ from the sample. The amplitude of the oscillating field is $\sim 3-5 \mathrm{G}$. The field gradient $d H_{z} / d z$ due to the coil is $\sim 500-750 \mathrm{G} / \mathrm{m}$, where the axis of the coil is along the $z$-direction; the transverse gradient is negligible.

[13] P. E. Wigen, in Magnetic Multilayers, edited by L. H. Bennett and R. E. Watson (World Scientific Publishing Co. Pte. Ltd., Singapore, 1994), pp. 183-226.

[14] C. P. Slichter, Principles of Magnetic Resonance (Springer-Verlag, New York, 1989).

[15] Digital Instruments, 520 E. Montecito St, Santa Barbara, CA. The experiments reported here use type ESP cantilevers which are $440 \mu \mathrm{m}$ long, 30-40 $\mu \mathrm{m}$ wide and 1-3 $\mu \mathrm{m}$ thick. 
[16] J. A. Sidles and D. Rugar, Phys. Rev. Lett. 70, 3506 (1993).

[17] R. Damon and J. Eshbach, J. Phys. Chem. Solids 19, 308 (1961).

[18] The $\mathrm{Nd}_{2} \mathrm{Fe}_{14} \mathrm{~B}$ particles were obtained from 3/8 inch rod cast at Ames Lab, Ames, Iowa. To ensure single phase material and improve the probability that each particle would consist of a single grain, the cast $\mathrm{Nd}_{2} \mathrm{Fe}_{14} \mathrm{~B}$ was homogenized by annealing for approximately 96 hours near the decomposition temperature. Small particles were obtained by crushing a section of the rod into a fine powder inside a glove box containing purified $\operatorname{Ar}\left(\mathrm{H}_{2} \mathrm{O}<1 \mathrm{ppm} ; \mathrm{O}_{2}<1 \mathrm{ppm}\right)$. Measurements were performed within two months of crushing. 


\section{FIGURES}

FIG. 1. A schematic diagram of the MRFM apparatus. The relevant cantilever vibration is in the $\mathbf{z}$-direction.

FIG. 2. The swept field ESR/MRFM spectrum from two particles of DPPH, one ( 7 nanograms) mounted above and one ( $\sim 3$ nanograms $)$ below the cantilever. The oscillation amplitude of the cantilever is shown as a function of distance, $z$, from the end of the bar magnet to the center of the sensitive slice where the resonance condition is satisfied. The negative value of the oscillation amplitude is due to the phase sensitive detection of the lock-in amplifier. The two antisymmetric dispersion signals from the two particles (whose centers are indicated by the arrows) are distinct, demonstrating rudimentary one-dimensional "imaging". The AFM cantilever is made

of pure $\mathrm{Si}$, about $440 \times 40 \times 3 \mu \mathrm{m}^{3}$ in size and has a loaded resonance frequency of $8.8 \mathrm{kHz}$ and a $Q$-value of 20,000 . The $\mathrm{rf}$ frequency is $828 \mathrm{MHz}$.

FIG. 3. An experimental FMR/MRFM spectrum of a microscopic $\left(20 \times 40 \times 3 \mu \mathrm{m}^{3}\right)$ single crystal YIG film which shows the nonresonance (NR) mode and a family of magneto-static modes (labeled $\left.m_{1}, m_{2}, \ldots\right)$. The centers of these magneto-static modes are indicated by the arrows as shown in the inset. The rf frequency is $825 \mathrm{MHz}$.

FIG. 4. The field of the $\mathrm{Nd}_{2} \mathrm{Fe}_{14} \mathrm{~B}$ particle. The sign and magnitude of component of the field (called $B_{M}^{\|}$) parallel to the applied bias field $B_{0}$ is shown. We show the value of the field at the center of the DPPH particle as a function of its distance $z$ to the nearest end of the $\mathrm{Nd}_{2} \mathrm{Fe}_{14} \mathrm{~B}$ particle. The solid lines are the theoretical fit assuming the $\mathrm{Nd}_{2} \mathrm{Fe}_{14} \mathrm{~B}$ particle has a cylindrical shape with a radius of $20 \mu \mathrm{m}$ and a length of $70 \mu \mathrm{m}$. Values of the magnetization $\mathcal{M}_{H}$ of 120 $\mathrm{emu} / \mathrm{cm}^{3}$ and $90 \mathrm{emu} / \mathrm{cm}^{3}$ were used in the fit for $\mathbf{B}_{0} \| \mathbf{M}$ and $\mathbf{B}_{0} \|-\mathbf{M}$ respectively.

FIG. 5. Variation of the cantilever resonance frequency $f_{c}$ with the applied bias field $B_{0}$. 
FIG. 6. (a) A schematic diagram of the cantilever depicting the forces between the polarized moment $\mathbf{M}$ of the $\mathrm{Nd}_{2} \mathrm{Fe}_{14} \mathrm{~B}$ particle and the bias field $\left(F_{\tau}\right)$ and the gradient of the field $\left(F_{g}\right)$. (b) A simplified model of (a) used to calculate the dependence of the cantilever frequency $f_{c}$ on $B_{0} . k$ is the cantilever spring constant. 